Article

\title{
Bicyclic Boronates as Potent Inhibitors of AmpC, the Class C $\beta$-Lactamase from Escherichia coli
}

\author{
Pauline A. Lang ${ }^{1} \mathbb{D}$, Anete Parkova ${ }^{2,+}$, Thomas M. Leissing ${ }^{1,+},{ }^{+}$Karina Calvopiña ${ }^{1}{ }^{(D)}$, \\ Ricky Cain ${ }^{3, \ddagger}$, Alen Krajnc ${ }^{1, \ddagger} \mathbb{D}$, Tharindi D. Panduwawala ${ }^{1} \mathbb{D}$, Jules Philippe ${ }^{4}$, \\ Colin W. G. Fishwick ${ }^{3}$, Peteris Trapencieris ${ }^{2}$, Malcolm G. P. Page ${ }^{4} \mathbb{D}$, \\ Christopher J. Schofield ${ }^{1, *(D)}$ and Jürgen Brem ${ }^{1, *(D)}$ \\ 1 Department of Chemistry, University of Oxford, Chemistry Research Laboratory, Oxford OX1 3TA, UK \\ Latvian Institute of Organic Synthesis, LV-1006 Riga, Latvia \\ School of Chemistry, University of Leeds, Leeds LS2 9JT, UK \\ 4 Jacobs University Bremen gGmbH, 28759 Bremen, Germany \\ * Correspondence: Christopher.schofield@chem.ox.ac.uk (C.J.S.); jurgen.brem@chem.ox.ac.uk (J.B.) \\ $\dagger$ These authors contributed equally. \\ $\ddagger$ These authors contributed equally.
}

Received: 30 April 2020; Accepted: 1 June 2020; Published: 12 June 2020

\begin{abstract}
Resistance to $\beta$-lactam antibacterials, importantly via production of $\beta$-lactamases, threatens their widespread use. Bicyclic boronates show promise as clinically useful, dual-action inhibitors of both serine- (SBL) and metallo- (MBL) $\beta$-lactamases. In combination with cefepime, the bicyclic boronate taniborbactam is in phase 3 clinical trials for treatment of complicated urinary tract infections. We report kinetic and crystallographic studies on the inhibition of AmpC, the class $C \beta$-lactamase from Escherichia coli, by bicyclic boronates, including taniborbactam, with different C-3 side chains. The combined studies reveal that an acylamino side chain is not essential for potent AmpC inhibition by active site binding bicyclic boronates. The tricyclic form of taniborbactam was observed bound to the surface of crystalline AmpC, but not at the active site, where the bicyclic form was observed. Structural comparisons reveal insights into why active site binding of a tricyclic form has been observed with the NDM-1 MBL, but not with other studied $\beta$-lactamases. Together with reported studies on the structural basis of inhibition of class A, B and D $\beta$-lactamases, our data support the proposal that bicyclic boronates are broad-spectrum $\beta$-lactamase inhibitors that work by mimicking a high energy 'tetrahedral' intermediate. These results suggest further SAR guided development could improve the breadth of clinically useful $\beta$-lactamase inhibition.
\end{abstract}

Keywords: antibiotic resistance; $\beta$-lactam antibacterial; bicyclic boronate inhibitors; VNRX-5133/taniborbactam; vaborbactam; metallo- and serine- $\beta$-lactamase inhibition; transition state analogue

\section{Introduction}

The $\beta$-lactams are amongst the most important antibiotics [1], but their widespread use is challenged by resistance, substantially through the global dissemination of $\beta$-lactamases $[2,3]$. There are two structural/mechanistic groups of $\beta$-lactamases-the nucleophilic serine- $\beta$-lactamases (SBLs: Ambler classes A, C, D) and the zinc dependent metallo- $\beta$-lactamases (MBLs: Ambler class B) (Figure 1b) [4]. Treatment of bacterial infections exhibiting resistance via some SBLs, particularly class A enzymes, was substantially advanced by the co-administration of a $\beta$-lactam antibiotic with a $\beta$-lactam-based SBL inhibitor, i.e., clavulanic acid [5,6], sulbactam [7], or tazobactam [8] (Figure 1a). However, these inhibitors are inactive, or are insufficiently active, against most class C SBLs, class 
A carbapenemases, and class B MBLs [3]. The clinical introduction of avibactam and avibactam derivatives such as relebactam [9] was an important step in more broadly combating SBLs because avibactam is active against class $A \beta$-lactamases (including carbapenemases), class $C \beta$-lactamases, and some class $D \beta$-lactamases [10]. Avibactam is also important because it demonstrates the clinical viability of non- $\beta$-lactam-based $\beta$-lactamase inhibition [11]. However, there is emerging SBL-mediated resistance to avibactam $[12,13]$ and MBLs can hydrolyze avibactam, albeit slowly [14], suggesting that its future use may be compromised by the evolution of new $\beta$-lactamases $[14,15]$.

(a)

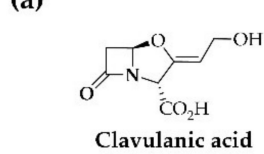

(b)

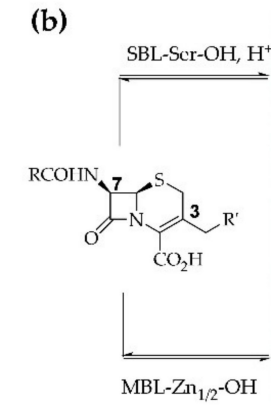

(c)

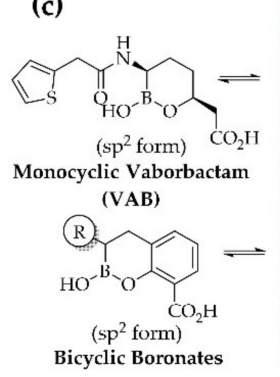

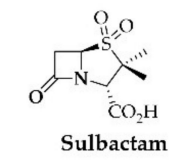
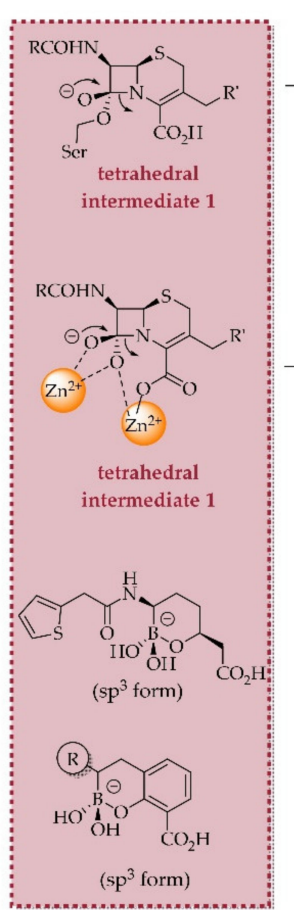
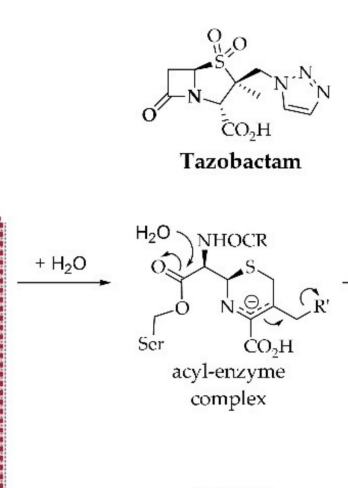
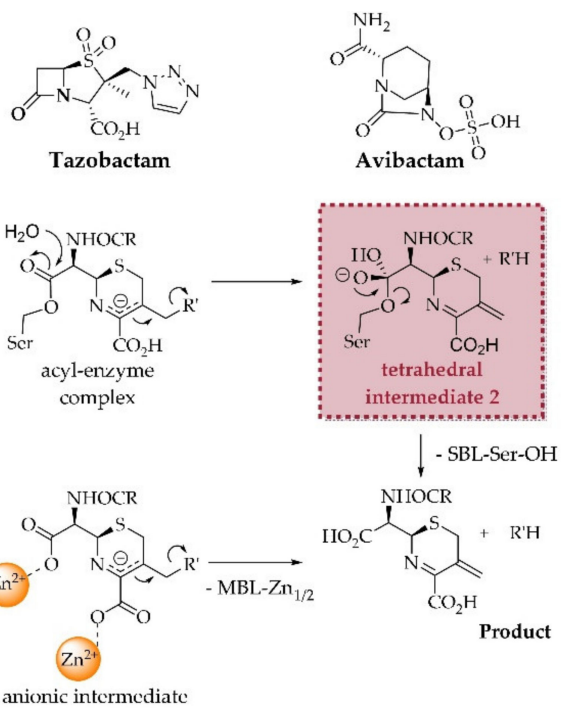

(d)

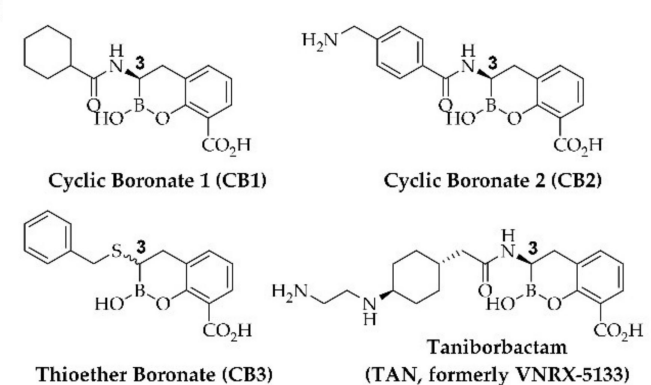

Figure 1. Mechanistic basis for $\beta$-lactamase inhibition by (bi)cyclic boronates. (a) clinically used $\beta$-lactamase inhibitors; (b) outline general mechanisms of SBLs and MBLs, exemplified by hydrolysis of a cephalosporin with elimination at C-3'. The tetrahedral intermediates, common to both SBL and MBL catalysis, may be mimicked by the $\mathrm{sp}^{3}$ form of (bi)cyclic boronates; (c) equilibria between $\mathrm{sp}^{2}$ - and $\mathrm{sp}^{3}$-hybridized forms of mono- and bi-cyclic boronates; (d) structures of bicyclic boronate $\beta$-lactamase inhibitors cyclic boronate 1 (CB1), cyclic boronate 2 (CB2), the thioether boronate (CB3), and taniborbactam (TAN, formerly VNRX-5133). Note that the CB3 used in this study contained a mixture of the $(3 S)$ - and (3R)-enantiomers ( 1:3, respectively) [16].

By contrast with the successes in inhibiting SBLs, to date, there are no clinically useful MBL inhibitors [3]. There is thus interest in development of non-acylating $\beta$-lactamase inhibitors, especially those exhibiting dual-action SBL and MBL inhibition. Boron-based compounds have long been known as SBL and, more recently, MBL inhibitors [17]. After early work on acyclic boronic acids, recent efforts have focused on cyclic boronates. One such compound, the (predominantly) monocyclic boronic acid vaborbactam [18] (VAB, Figure 1c) which is approved for treatment of complicated urinary tract infections (cUTI) in co-administration with meropenem [19]. Vaborbactam is a relatively potent inhibitor of class A ESBLs and the KPC carbapenemases, but is not, at least usefully, active against tested MBLs and clinically relevant class C and D SBLs [19]. By contrast, boronates that are predominantly 
bicyclic in solution, are able to inhibit many, but not all, of the tested representatives of all four Ambler $\beta$-lactamase classes $[20,21]$. The bicyclic boronates are proposed to work by binding in a manner analogous to that of the tetrahedral intermediate(s) likely involved in $\beta$-lactam catalyzed hydrolysis by both SBLs and MBLs [20,21] (Figure 1b). Taniborbactam (TAN, formerly VNRX-5133, Figure 1d) has passed phase 1 clinical testing [22] and is currently in phase 3 clinical testing in combination with cefepime (registration No. NCT03840148 at ClinicalTrials.gov). TAN has been shown to successfully inhibit SBLs and MBLs in vitro [23-26], and to restore cefepime's activity in vivo against a class A SBL (CTX-M-14) producing strain of Klebsiella pneumoniae in a neutropenic lung model of infection as well as CTX-M-15 expressing E. coli in ascending urinary tract infections in mice [26].

Many $\beta$-lactamases are encoded by plasmids, but the AmpC type $\beta$-lactamases that are present in most Escherichia coli strains are chromosomally encoded [27]. While normally expressed at low levels, mutations in the $a m p C$ gene promoter and/or attenuator regions can lead to constitutive hyperproduction of AmpC in E. coli [28,29]. This is clinically relevant because E. coli is a common Gram-negative pathogen, which is often responsible for bloodstream infections [30].

Structural data on the modes of action of bicyclic boronate $\beta$-lactamase inhibitors are relatively limited [20,21,24,26,31-33]. Work to date indicates that more than one side chain binding mode can occur, making structure-guided optimization challenging. Work on the B1 subfamily MBL NDM-1 has shown partial cyclisation of the TAN acylamino side chain to form a tricyclic structure, as an observed bound at the NDM-1 active site by protein crystallography [24]. The extent to which such tricyclisation contributes to $\beta$-lactamase inhibition more generally is unclear.

To enable future efforts on the optimization of bicyclic boronates, we report crystallographic and kinetic studies on inhibition of the clinically important class C AmpC $\beta$-lactamase from Escherichia coli $\left(\mathrm{AmpC}_{E C}\right)$ by TAN, a previously reported model bicyclic boronate 2 (CB2) [20,21,34], and a thioether bicyclic boronate derivative (CB3) that we have recently shown to have enhanced potency against some SBLs and MBLs in vitro [16,35]. Together with previously reported studies $[20,21,24,26,31-33]$, the crystallographic results support the proposal that bicyclic boronates mimic the tetrahedral intermediate(s) common to both SBL and MBL catalysis. The results indicate scope for the modification of the C-3 side chain of bicyclic boronates to improve potency towards AmpC type $\beta$-lactamases and inform on the likelihood of tricycle formation.

\section{Materials and Methods}

\subsection{Materials}

CB2, TAN, and CB3 were prepared as previously described $[16,20,24]$; VAB was purchased from Cayman Chemical (An Arbor, MI, USA). FC-5 was prepared as previously described [36].

\subsection{Enzyme Production}

Recombinant AmpC from Escherichia coli was produced according to a modification of the reported protocol [37]. In brief, $\mathrm{AmpC}_{E C}$ was produced using the pAD7 vector [38] in E. coli W3110 cells using 2TY media supplemented with $12.5 \mathrm{mg} / \mathrm{mL}$ tetracycline. Cells were grown overnight at $37^{\circ} \mathrm{C}$, harvested via centrifugation $\left(10 \mathrm{~min}, 12,000 \times g, 4^{\circ} \mathrm{C}\right)$, resuspended in $50 \mathrm{~mL}$ lysis buffer $(20 \mathrm{mM}$ Tris $\mathrm{pH}$ 7.5, $10 \mathrm{mM} \mathrm{MgCl} 2,50 \mu \mathrm{g} / \mathrm{mL}$ DNAse I), then lyzed via sonification. Subsequent chromatography steps employed ÄKTA (GE Healthcare, Chicago, IL, USA) FPLC machines. The resultant supernatant was dialyzed with three buffer exchanges of $300 \mathrm{~mL} 10 \mathrm{mM}$ Tris pH 6.75, then loaded onto a SP Sepharose (GE Healthcare, Chicago, IL, USA) cation exchange column and gradually eluted using a gradient of 10-75 mM Tris $\mathrm{pH}$ 7.0. Fractions containing the purified protein were pooled and concentrated to $20 \mathrm{mg} / \mathrm{mL}$ using an Amicon Ultra (MilliporeSigma, Burlington, MA, USA) centrifugal filter (15 mL, $10 \mathrm{kDa}$ MWCO). The protein was highly purified (>95\%) as judged by SDS-PAGE and electrospray ionization mass spectrometry analyses (observed mass: 39,551 Da, calculated mass: 39,551 Da). 


\subsection{Kinetics}

All kinetic measurements were carried out in competition assays using the fluorescent substrate FC-5 [36] under steady-state conditions monitoring hydrolysis, in $100 \mathrm{mM}$ phosphate buffer pH 7.5 supplemented with 0.01\% (v/v) Triton X-100 (Assay Buffer). Fluorescence was measured using a PHERAstarFS (BMG Labtech, Aylesbury, United Kingdom) plate-reader, recording emission spectra at $\lambda_{\mathrm{ex}}=380 \mathrm{~nm}$ and $\lambda_{\mathrm{em}}=460 \mathrm{~nm}$. IC 50 s were determined after 10 min incubation time of $500 \mathrm{pM}$ $A m p C_{E C}$ with varying concentrations of inhibitors and assayed using $5 \mu \mathrm{M}$ FC-5. Nonlinear regression analyses were carried out using GraphPad Prism V. 5.04 (GraphPad Software, San Diego, CA, USA).

As described for avibactam inhibition [11], the kinetics of bicyclic boronate inhibition of $\beta$-lactamases can be described assuming a two-step, reversible inhibition model:

$$
E+I \underset{k-1}{\stackrel{k_{1}}{\rightleftarrows}} E: I \underset{k-2}{\stackrel{k_{2}}{\rightleftarrows}} E-I
$$

with $E$ : enzyme; $I$ : inhibitor; $k_{1}$ : association rate constant; $k_{-1}$ : dissociation rate constant; $k_{2}$ : binding rate constant, and $k_{-2}$ : recyclization rate constant.

To obtain the pseudo first-order rate constant $k_{\mathrm{obs}}$ and the apparent inhibition constant $K_{\text {iapp, }}$ the rate of FC-5 $(5 \mu \mathrm{M})$ hydrolysis by $\operatorname{AmpC}_{E C}(100 \mathrm{nM})$ was measured in the presence of varying concentrations of the inhibitor. Time-courses were then first fitted to Equation (2) to give the observed initial rate constants $k_{\mathrm{obs}}$ as previously described [39]:

$$
P=V_{s} t+\left(V_{0}-V_{S}\right) \frac{\left(1-e^{-k_{o b s} t}\right)}{k_{o b s}}+P_{0}
$$

with $P$ : formed product, proportional to fluorescence signal; $P_{0}$ : initial fluorescence; $V_{S}$ : velocity of no-inhibitor control; and $V_{0}$ : velocity of no-enzyme control to estimate a fully inhibited enzyme.

Plotting the resultant $k_{\text {obs }}$ values against the inhibitor concentration [I] (Figure S1b) and fitting to Equation (3) gives the apparent second-order rate constant $\left(k_{2} / K^{\prime}\right)$

$$
k_{o b s}=k_{2}+\frac{k_{2}}{K^{\prime}} *[I]
$$

with $K^{\prime}$ representing the equilibrium coefficient of $A m p C_{E C}$ inhibition in the presence of FC-5 in the set concentration of $5 \mu \mathrm{M}$.

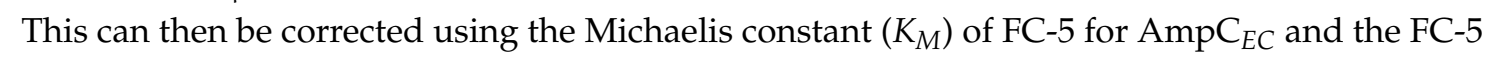
concentration [S] according to Equation (4) to give the second-order rate constant $\left(k_{2} / K\right)$ :

$$
\frac{k_{2}}{K}=\frac{k_{2}}{K^{\prime}} *\left(\frac{[S]}{K_{M}}+1\right)
$$

The same data were used to determine the apparent inhibition constant $K_{\text {iapp }}$ [40]. The reciprocals of the initial rates were plotted against inhibitor concentration (Figure S1a), giving a straight line for which the value of the intercept divided by the slope gives $K_{\text {iapp }}{ }^{\prime}$. From this, $K_{\text {iapp }}$ can be obtained after correction with the $K_{M}$ for FC-5 according to Equation (5):

$$
K_{\text {iapp }}=\frac{K_{\text {iapp }}^{\prime}}{1+\left(\frac{[S]}{K_{M}}\right)}
$$

Off rates ( $k_{-2}$ or $\left.k_{\text {off }}\right)$ were measured using the jump-dilution method [41] (Figure S1c). AmpC $E C$ $(1 \mu \mathrm{M})$ was incubated with the respective inhibitor $(10 \mu \mathrm{M}$ for TAN, CB2 and CB3, and $100 \mu \mathrm{M}$ for VAB) for $30 \mathrm{~min}$ at room temperature, then diluted 100,000 fold in the Assay Buffer (final enzyme concentration: $10 \mathrm{pM}$ ) and immediately assayed with $25 \mu \mathrm{M}$ FC-5. The data were fitted to Equation (2) with $V_{0}$ in this case representing the velocity of the no-enzyme control and $\mathrm{V}_{\mathrm{S}}$ representing the initial velocity of uninhibited enzyme. 
The half-life of the enzyme-inhibitor complex $t_{1 / 2}$ is given by Equation (6):

$$
t_{1 / 2}=\frac{\ln (2)}{k_{o f f}}
$$

\subsection{Antimicrobial Susceptibility Testing}

Minimum inhibitory concentrations (MICs) were determined by broth microdilution in triplicate and interpreted using published Clinical and Laboratory Standards Institute (CLSI) guidelines [22].

Ceftazidime (CAZ) was tested alone $\left(0.25-256 \mu \mathrm{g} \mathrm{mL}^{-1}\right)$ against DH5 $\alpha$ Escherichia coli and DH5 $\alpha$ E. coli containing the $\mathrm{pAD7}-\mathrm{AmpC} \mathrm{C}_{E C}$ plasmid and in combination with $\mathrm{CB} 2, \mathrm{CB}$, TAN, and VAB (all tested at a fixed concentration of $4 \mu \mathrm{g} \mathrm{mL} \mathrm{m}^{-1}$ ) using the $\mathrm{DH} 5 \alpha$ E. coli strain containing the $\mathrm{pAD} 7-\mathrm{AmpC}_{E C}$ plasmid [38].

\subsection{Crystallization Experiments, X-Ray Data Collection and Processing}

Crystallization plates (low reservoir Intelli-Plate 93-3, Art Robbins Instruments, Sunnyvale, CA, USA) were set up with a Phoenix RE Drop setter instrument (Art Robbins Instrument, Sunnyvale, CA, USA). Crystals were grown via the vapor diffusion technique at room temperature. To obtain $A m p C_{E C}$-TAN and $\mathrm{AmpC}_{E C}-\mathrm{CB} 3$ complex crystals, crystals of apo-AmpC $C_{E C}$ were grown in Condition A, comprising $200 \mathrm{~nL} \mathrm{AmpC} \mathrm{EC}_{\mathrm{EC}}(20 \mathrm{mg} / \mathrm{mL}$ in $50 \mathrm{mM}$ Tris $\mathrm{pH}$ 7.5), mixed with $200 \mathrm{~nL}$ Precipitant Solution (10 mM zinc chloride, $100 \mathrm{mM}$ MES pH 6.0 and 20\% (w/v) PEG 6000). Crystals grew at room temperature over $2-3$ days, were transferred into a well solution supplemented with approximately $20 \mathrm{mM}$ inhibitor and incubated for 15 and $10 \mathrm{~min}$, respectively. These $\mathrm{AmpC}_{\mathrm{EC}}$ crystals are highly sensitive to DMSO and prolonged soaking times ( $>15 \mathrm{~min}$ ). Soaking was successful for TAN and $\mathrm{CB} 3$, but, in the case of $\mathrm{CB} 2$, only low occupancy was observed for $\mathrm{CB} 2$ following soaking under the same conditions. A single crystal of the $\mathrm{AmpC}_{E C}-\mathrm{CB} 2$ complex was obtained via co-crystallization

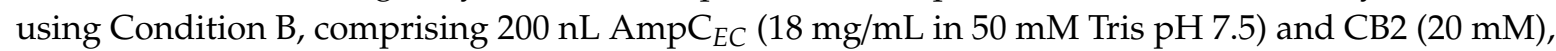
mixed with $200 \mathrm{~nL}$ Precipitant Solution (150 mM HEPES, 60\% (v/v) 2-methyl-2,4-pentanediol (MPD)). Crystals grew at room temperature over 1-2 weeks. Due to the less robust crystallization system and the lower resolution of the data obtained (see Table S1), the co-crystallization approach was not explored for TAN and CB3. Crystals were cryo-cooled and then stored in liquid nitrogen. Datasets from single crystals were collected using the i03 and i24 MX beamlines at the Diamond Light Source (Table S1). Structures were solved by molecular replacement in Phaser [42] using PDB ID 1IEM [43] as the starting model. Alternating cycles of refinement using PHENIX [44] and model building using Coot [45] were performed until $R_{\text {work }}$ and $R_{\text {free }}$ converged. Coordinates and structure factors have been deposited in the Protein Data Bank. PDB IDs are 6T3D, 6YEO, 6YPD and 6YEN for the crystal structure of AmpC from Escherichia coli in its apo-form and in complex with CB2, CB3, and TAN, respectively.

\section{Results and Discussion}

\subsection{Kinetic Studies of $A m p C_{E C}$ Inhibition by Bicyclic Boronates}

To investigate the importance of different side chains at the C-3 position (equivalent to C-6 of penicillins or C-7 of cephalosporins) of the bicyclic boronate core for inhibition of $\mathrm{AmpC}_{E C}$, steady-state kinetic assays (Table 1) were carried out using the fluorescent reporter substrate FC-5 [36]. All three tested bicyclic boronates showed enhanced potency for $\mathrm{AmpC}_{E C}$ inhibition compared to the monocyclic boronate $\mathrm{VAB}\left(K_{\text {iapp }} \sim 1-4 \mu \mathrm{M}\right.$ for the bicyclic boronates, compared to $19 \mu \mathrm{M}$ for VAB). Interestingly, the thioether $\mathrm{CB} 3$ was slightly more potent against isolated $\mathrm{AmpC}_{E C}$ than TAN and the structurally similar compound CB2 ( $K_{\text {iapp }} 1 \mu \mathrm{M}$ for $\mathrm{CB} 3$, compared to $3 \mu \mathrm{M}$ and $4 \mu \mathrm{M}$ for $\mathrm{CB} 2$ and TAN, respectively). The increased potency of CB3 likely results from accelerated binding $\left(k_{2} / K \sim 224 \times 10^{3} \mathrm{M}^{-1} \mathrm{~s}^{-1}\right.$ compared to $\sim 81 / 86 \times 10^{3} \mathrm{M}^{-1} \mathrm{~s}^{-1}$ for CB2/TAN and $\sim 7 \times 10^{3} \mathrm{M}^{-1} \mathrm{~s}^{-1}$ for VAB). However, dissociation of CB3 is equally accelerated ( $21 \times 10^{-3} \mathrm{~s}^{-1}$, compared to $3 \times 10^{-3} \mathrm{~s}^{-1}$ for CB2/TAN and $0.7 \times 10^{-3} \mathrm{~s}^{-1}$ for VAB), resulting in a shorter half-life of the enzyme-inhibitor complex for CB3. This proposal is supported by 
$\mathrm{pIC}_{50}$ measurements showing equivalent potency of all three tested bicyclic boronates after a $10 \mathrm{~min}$ pre-incubation period with $\mathrm{AmpC}_{E C}\left(\mathrm{pIC}_{50} \mathrm{~s}\right.$ of 7.5 for $\mathrm{CB} 2$, TAN, and $\mathrm{CB} 3$ compared to 6.3 for VAB).

Table 1. Kinetic analyses of $A m p C_{E C}$ inhibition by (bi)cyclic boronates in vitro. $K_{\text {iapp }}$ values and pseudo first-order rates $\left(k_{2} / K\right)$ were determined by assaying $100 \mathrm{nM} \mathrm{AmpC} \mathrm{CC}_{\mathrm{C}}$ with $5 \mu \mathrm{M}$ FC-5 [36]. $k_{\text {off }}$ rates were determined after jump-dilution (100,000 fold) of $\operatorname{AmpC}_{E C}(1 \mu \mathrm{M})$ that had been pre-incubated with cyclic boronates (10 $\mu \mathrm{M}$ for TAN, CB2 and CB3 and $100 \mu \mathrm{M}$ for VAB) at room temperature for $30 \mathrm{~min}$, then assayed using $25 \mu \mathrm{M}$ FC-5. $\mathrm{pIC}_{50}$ s were obtained from assays using $500 \mathrm{pM} \mathrm{AmpC} E C$ and $5 \mu \mathrm{M}$ FC-5 following a $10 \mathrm{~min}$ inhibitor pre-incubation at room temperature. Buffer: $50 \mathrm{mM}$ Tris, $\mathrm{pH} 7.5,0.01 \%(v / v)$ Triton X-100. Data were analyzed as described in Materials and Methods.

\begin{tabular}{cccccc}
\hline Inhibitor & $\boldsymbol{K}_{\text {iapp }}(\boldsymbol{\mu M})$ & $\boldsymbol{k}_{2} / \mathbf{K}\left(\mathbf{M}^{-\mathbf{1}} \mathbf{s}^{\mathbf{- 1}}\right) \times \mathbf{1 0}^{\mathbf{3}}$ & $\boldsymbol{k}_{\text {off }}\left(\mathbf{s}^{-\mathbf{1}}\right) \times \mathbf{1 0}^{-\mathbf{3}}$ & $\mathbf{t}_{\mathbf{1 / 2}}(\mathbf{m i n})$ & $\mathbf{p I C}_{\mathbf{5 0}}$ \\
\hline VAB & $19.1 \pm 1.32$ & $6.55 \pm 0.46$ & $0.69 \pm 0.03$ & $16.8 \pm 0.7$ & $6.32 \pm 0.03$ \\
TAN & $3.73 \pm 0.50$ & $85.6 \pm 9.2$ & $2.54 \pm 0.51$ & $4.55 \pm 0.91$ & $7.53 \pm 0.02$ \\
CB2 & $3.26 \pm 0.40$ & $81.0 \pm 6.4$ & $2.77 \pm 0.27$ & $4.18 \pm 0.41$ & $7.49 \pm 0.01$ \\
CB3 & $1.16 \pm 0.14$ & $224 \pm 21$ & $20.9 \pm 12.8$ & $0.55 \pm 0.34$ & $7.53 \pm 0.02$ \\
\hline
\end{tabular}

\subsection{Microbiology Experiments Confirm Potential of (Bi)cyclic Boronates to Inhibit AmpC $C_{E C}$ in Cells}

We then investigated the activity of VAB, TAN, CB2 and CB3 (fixed at $4 \mu \mathrm{g} \mathrm{mL}{ }^{-1}$ ) in combination with ceftazidime (CAZ) against $E$. coli $\mathrm{DH} 5 \alpha$ producing $\mathrm{AmpC}_{E C}$ (Table 2). Production of $\mathrm{AmpC}_{E C}$ substantially increased the extent of CAZ resistance (MIC $256 \mu \mathrm{g} \mathrm{mL}^{-1}$ compared to $1 \mu \mathrm{g} \mathrm{mL} \mathrm{m}^{-1}$ without the $A m p C_{E C}$ expressing plasmid); all the tested inhibitors significantly restored CAZ activity against the $A_{m p C} C_{E C}$ expressing strain. Despite the high potency of CB3 observed in the kinetic analyses against isolated $A m p C_{E C}$, it was less potent than $C B 2$, TAN or VAB in restoring CAZ activity in the engineered strain, i.e., CB3 reduced the MIC only to $4 \mu \mathrm{g} \mathrm{mL} L^{-1}$, whereas the other inhibitors restored the activity of $\mathrm{CAZ}$ to $\leq 1 \mu \mathrm{g} \mathrm{mL} \mathrm{m}^{-1}$.

Table 2. Microbiology analyses with vaborbactam (VAB), taniborbactam (TAN), cyclic boronate 2 (CB2) and cyclic boronate 3 (CB3). All inhibitors were tested at a fixed concentration of $4 \mu \mathrm{g} \mathrm{mL} \mathrm{m}^{-1}$.

\begin{tabular}{lcccccc}
\hline & & \multicolumn{5}{c}{$\begin{array}{c}\text { Minimum Inhibitory Concentration } \\
\text { Ceftazidime }\left(\mu \mathbf{g L}^{-\mathbf{1}}\right)\end{array}$} \\
\cline { 3 - 7 } Strain & Plasmid & CAZ & CAZ (+ VAB) & CAZ (+ TAN) & CAZ (+ CB2) & CAZ (+ CB3) \\
\hline DH5 $\alpha$ & - & 1 & - & - & - & - \\
DH5 $\alpha$ & $\mathrm{pAD7}$ & 256 & 1 & 1 & 0.5 & 4 \\
\hline
\end{tabular}

\subsection{Amp $C_{E C}$ Crystal Structures with Bicyclic Boronates Give New Insights into Structural Basis of Inhibition}

To investigate the structural basis of $\mathrm{AmpC}_{E C}$-inhibition by the three bicyclic boronates $(\mathrm{CB} 2, \mathrm{TAN}$, and $\mathrm{CB} 3$ ), we obtained crystal structures of their complexes with recombinant $A m p C_{E C}$. The structures

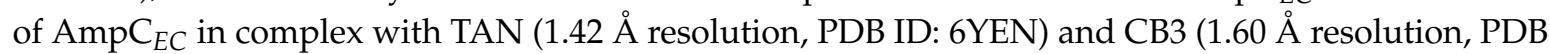
ID: 6YPD) were obtained by soaking of apo-AmpC $C_{E C}$ crystals. A structure of the complex of $A m p C_{E C}$ with CB2 (2.03 Å resolution, PDB ID: 6YEO) was obtained by co-crystallization (see Materials and Methods). Apo-AmpC $E_{E C}$ crystallized with a single chain in the asymmetric unit (space group $\mathrm{P}_{3} 32$ ), while the asymmetric unit of the $\mathrm{AmpC}_{E C}-\mathrm{CB} 2$ co-crystal complex contains four molecules of the $\beta$-lactamase (space group P222 1 ).

The overall fold of the $\mathrm{AmpC}_{E C}$-boronate complexes is very similar to that of the apo-AmpC $E C$ structure (PDB ID: 6T3D; main chain RMSDs with respect to the apo-structure: $0.39,0.15,0.07 \AA$ for $\mathrm{CB} 2$, TAN, and CB3, respectively, for chain A). In all three structures, analysis of the electron density maps for the $\mathrm{AmpC}_{E C}$ active site indicates that the bicyclic boronates CB2, TAN, and CB3 have reacted with the nucleophilic S64 to form an anionic tetrahedral species in which the boron is $\mathrm{sp}^{3}$ hybridized (Figure 2), as observed for other bicyclic boronate SBL complex structures $[20,21,26,31-33]$. The bicyclic 
core of all three inhibitors in complex with $\mathrm{AmpC}_{E C}$ is bound in a similar manner to that observed for cyclic boronate 1 (CB1, Figure 1d) in complex with the Pseudomonas aeruginosa AmpC (PDB ID: $6 \mathrm{I} 30$ [32]). In none of these cases did any of the active site bound bicyclic boronates form a tricyclic structure, as was observed in crystallographic studies of the MBL NDM-1-TAN complex [24].

(a)

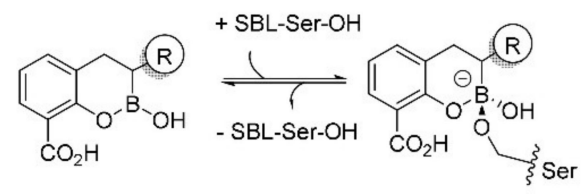

Bicyclic Boronates $\left(\mathrm{sp}^{2}\right.$ form)

(c)

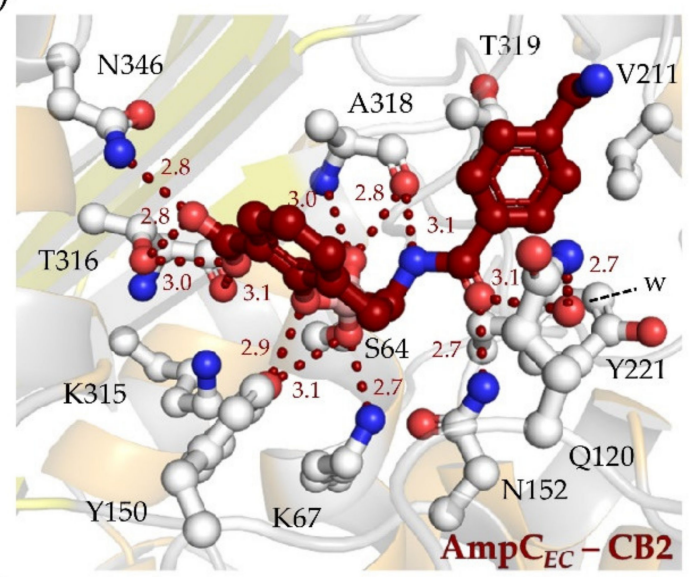

(e)

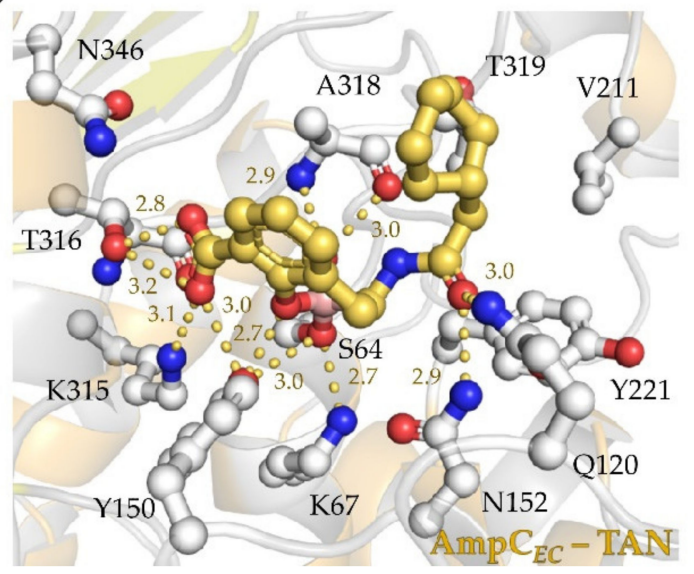

(b)

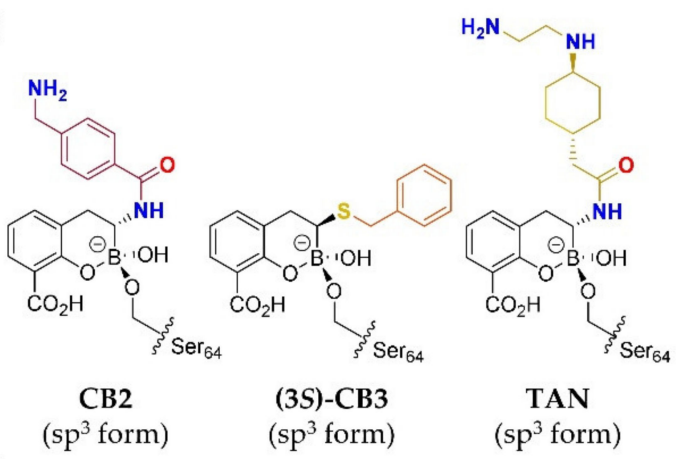

(d)

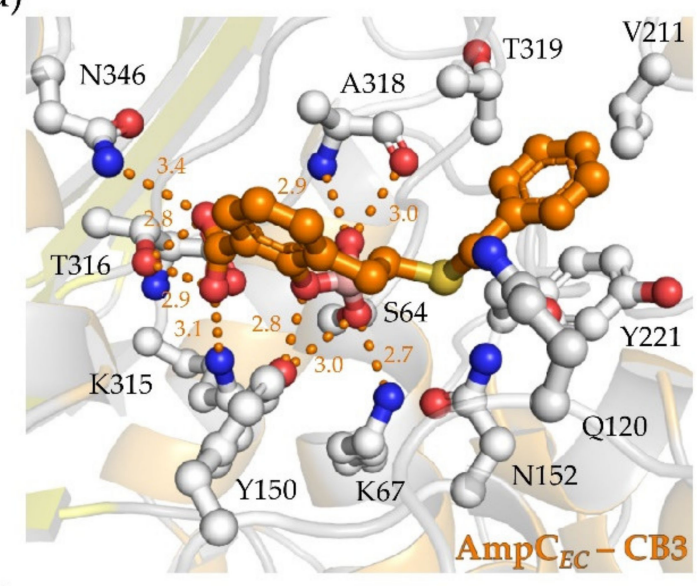

(f)

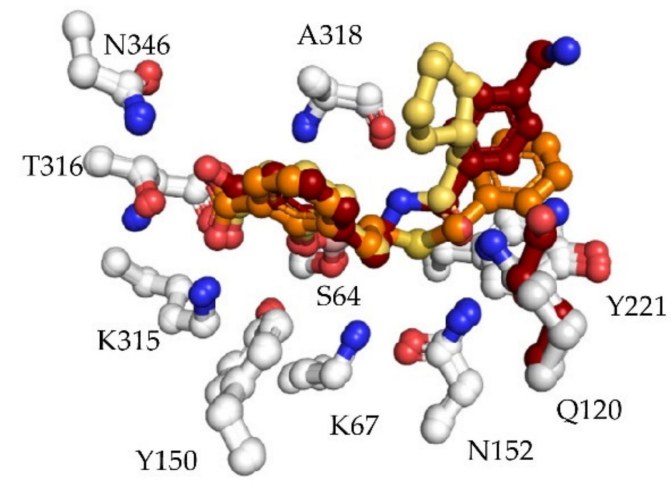

Figure 2. Structural basis for $\mathrm{AmpC}_{E C}$ inhibition by bicyclic boronates. (a) reaction between $\mathrm{sp}^{2}$-hybridized bicyclic boronates and SBLs give a serine-bonded anionic $\mathrm{sp}^{3}$-hybridized species; (b) structures of serine-bonded $\mathrm{sp}^{3}$ forms of the bicyclic boronates; (c-e) active site views of complexes of $\mathrm{AmpC}_{E C}$ with $\mathrm{CB} 2, \mathrm{CB} 3$, and TAN. Hydrogen-bonding interactions are shown in colored dashes, distances are in $\AA$; (f) overlay of active site views, color coding as in (c-e). Note that the Q120 side chain is rotated in $\mathrm{AmpC}_{E C}-\mathrm{CB} 2$ structure (highlighted in dark red); it is unclear whether this is induced by binding of $\mathrm{CB} 2$, or due to the crystallization conditions (see text). 
The aryl carboxylic acid of the bicyclic boronates is positioned similarly to that of the monocyclic inhibitor VAB as observed in complex with the Enterobacter cloacae AmpC (PDB ID: 4XUX [18]) and that of CB1 observed in complex with P. aeruginosa AmpC [32]. The aryl carboxylic acid of all the bicyclic boronates is involved in hydrogen-bonding or electrostatic interactions with active site residues K315, T316, and N346. The boron-linked hydroxyl group engages in hydrogen-bonding interactions with the backbone NH and oxygen of A318, as observed for the equivalent S345/338 residues in the P. aeruginosa AmpC-CB1 and E. cloacae AmpC-VAB complex structures [18,32].

As observed in the P. aeruginosa AmpC-avibactam [46] and P. aeruginosa AmpC-relebactam [9,47] complex structures, the amide nitrogen and carbonyl group of the TAN acylamino side chain are positioned to make hydrogen-bonding interactions with active site residues, i.e., Q120, N152, and A318. The cyclohexyl ring of TAN is directed out of the active-site R1 pocket (Figure 2e); only weak density was observed for the likely disordered terminal alkyl-amine side chain, which was excluded from the model.

$\mathrm{AmpC}_{E C}$ was co-crystallized with CB2 giving crystals with four molecules in each asymmetric unit, all of which manifested clear and continuous electron density for the covalently bound CB2 at the active site (Figure S2). The overall folds and orientations of active site residues and CB2 (Figure S2d) are very similar in all four chains (main chain RMSD of Chain B, C and D in relation to chain A: 0.31, 0.28 , and $0.33 \AA$, respectively). Binding of the bicyclic boronate core of $C B 2$, together with that of the amido side chain group portion, is very similar to that observed for TAN (CB3 has a different type of side chain).

Interestingly, in the CB2 structure, the side chain of Q120 is directed away from the active site in all four molecules in the asymmetric unit (Figure 2c); Q120 interacts with a water molecule (Figure 2c, ' $\mathrm{w}$ ', observed in all chains of the ASU), which in turn interacts with the carbonyl oxygen of the acylamino CB2 side chain. In the apo structure of $A m p C_{E C}$ in condition A (PDB ID: 6T3D), the Q120 side chain is oriented towards the active site; however, the apparently weak density and elevated B-factors for the Q120 side chain indicate partial disorder. The reorientation of the Q120 side chain relative to the apo-enzyme observed in the $\mathrm{AmpC}_{E C}-\mathrm{CB} 2$ complex is not manifest in the structure of the $A m p C_{E C}-T A N$ complex. Although the AmpC $C_{E C}-C B 2$ complex was co-crystallized using a different crystallization condition than for the two other structures reported here (see Materials and Methods), because the Q120 side chain does not interact with residues of other $\mathrm{AmpC}_{E C}$ molecules in the crystalline lattice, reorientation due to formation of crystal contacts seems unlikely. In a crystal structure of $\mathrm{AmpC}_{E C}$, obtained by soaking with CB2, partial density for the bound CB2 was observed in the active site, though reorientation of the Q120 side chain was not observed (data not shown, because of poor electron density for the CB2 C-3 side chain). It is thus unclear whether the different orientation of Q120 compared to apo-AmpC $E C$ is due to the different crystallization conditions or due to a specific feature of CB2 binding. The positioning of the Q120 side chain could impact on the potency of inhibition by bicyclic boronates containing an amido side chain, as in CB2 and TAN.

A structure of the AmpC $C_{E C}-\mathrm{CB} 3$ complex was obtained by soaking of apo crystals of the $\beta$-lactamase. CB3 does not contain the amido $C-3$ side chain, but instead features a benzyl-thioether side chain (Figure 1d) [16]. CB3 was synthesized as a mixture of the (3S)- and (3R)-isomers with the (3R)-isomer in excess ( 24:76 (S:R) ratio) [16]. Although binding of low levels of the (3R)-isomer cannot be excluded, only evidence for binding of the (3S)-CB3 was observed at the active site of $A m p C_{E C}$. The thioether side chain is partially disordered, as manifested by $\sim 50 \%$ elevated B-factors compared to the CB3 average. The thioether is positioned to make hydrophobic $\pi$-stacking interaction with Y221( $4 \AA$ distance between Y221 and CB3 benzyl rings) and hydrophobic interactions with the side chains of V211 and T319 in the R1 pocket (Figure 2d). These hydrophobic residues located on the $\mathrm{AmpC}_{E C}$ $\Omega$-loop (residuess 185-225) are also present in the P. aeruginosa AmpC, rationalizing the high potency of CB3 against both $\mathrm{AmpC}_{E C}$ and P. aeruginosa AmpC and likely other class C SBLs, as reported here and elsewhere [16]. Notably, mutations in the $\Omega$-loop, e.g., deletion of Y221, have been observed to widen the substrate specificity and also confer ceftazidime-avibactam resistance [12]. Such AmpC variants 
may be less susceptible to bicyclic boronates like CB3 and are thus a potential source of resistance to such inhibitors. This is of particular concern given that mutations in the $\Omega$-loop have been linked to reduced susceptibility of cefepime against AmpC-expressing E. coli [48], which is the $\beta$-lactam proposed for use in combination with TAN and which is not efficiently turned-over by the wildtype $\mathrm{AmpC}_{E C}$.

Interestingly, apart from covalent modification of S64, analysis of the electron density map in the structure obtained by soaking $A m p C_{E C}$ with TAN clearly reveals a second TAN molecule at the interface between chain A and symmetry related molecules (Figure S3a). The second TAN molecule adopts a U-shaped conformation in which its side chain folds over the bicyclic core (Figure 3); it is positioned to form hydrogen-bonding interactions with multiple water molecules that interact with the backbone carbonyls of P140, A141, and N102, and to make hydrophobic interactions with the side chains of A143 and A98 in a symmetry related molecule (Figure 3a).

(a)

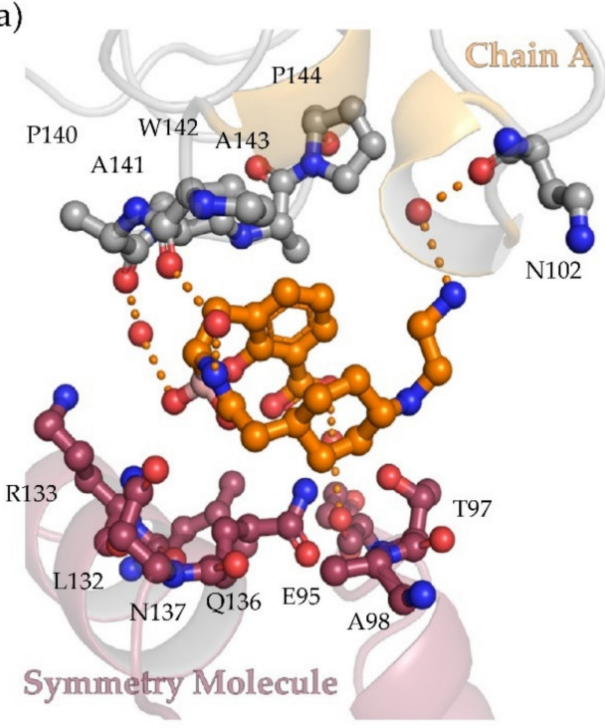

(b)

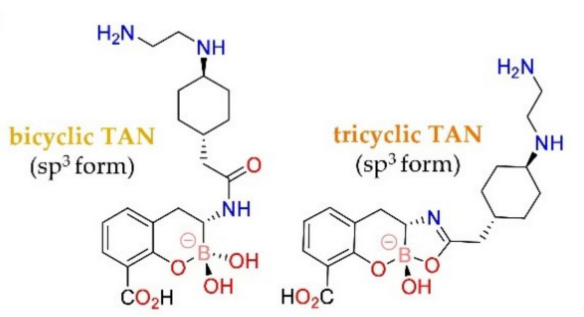

(c)

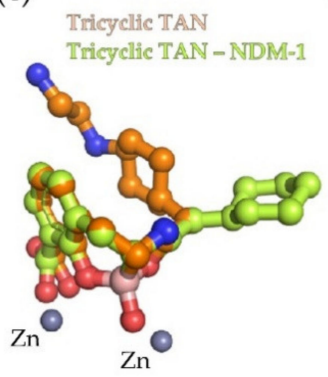

(d)

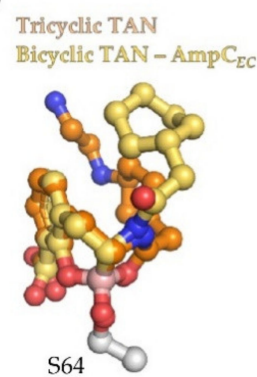

Figure 3. Comparison of bi- and tri-cyclic forms of $\mathrm{sp}^{3}$ hybridized taniborbactam in complex with $A m p C_{E C}$. (a) The tricyclic form of TAN as observed at the interface between symmetry related $\mathrm{AmpC}_{E C}$ molecules along a three-fold rotation axis (see Figure S3a for overview, PDB ID 6YEN). Residues within $6 \AA$ of TAN are shown as grey/purple sticks, hydrogen-bonding interactions as orange dashes, and waters as red spheres; (b) structures of proposed bi- and tricyclic $\mathrm{sp}^{3}$ forms of TAN; (c) overlay of the 'interface' tricyclic TAN (AmpC $C_{E C}$, orange) with active site bound tricyclic TAN (NDM-1, green); (d) overlay of the 'interface' tricyclic TAN (AmpC $E C$, orange) with active site bound bicyclic TAN bonded to $\mathrm{AmpC}_{E C} \mathrm{~S} 64$ (yellow). Note, with the MBL NDM-1 (PDB ID: 6RMF), both the bicyclic and the tricyclic forms were observed in one chain of the crystallographic dimer (chain A, shown here), while, in the second chain, only the tricyclic form was observed [24].

Notably, with the surface bound TAN molecule, its C-3 amido side chain is rotated relative to the conformation observed for bicylic TAN at the active site, positioning the carbonyl derived oxygen atom in close proximity to the boron. Based on the short distance between the refined carbonyl oxygen and the boron $(1.5 \AA)$, this molecule of TAN was thus modelled in a tricyclic form, as shown in Figure $3 \mathrm{~b}$. The 5-membered ring of this TAN molecule was modelled in its unsaturated form based on its planar conformation and the angle between the carbonyl-derived oxygen, carbon- and amide-derived nitrogen atoms $\left(122^{\circ}\right)$. The observation of tricyclic TAN on the surface of $A m p C_{E C}$ indicates the viability of its formation in solution; reaction of acyl-amino side chains to form a ring onto a proximate boronic acid has synthetic precedent [49]. An analogous tricycle has also been observed in the complex formed between the B1 MBL NDM-1 and TAN [24]. Overlay of the tricyclic boronate core 
observed with $\mathrm{AmpC}_{E C}$ and that observed in the active site of NDM-1 shows a strong resemblance (Figure 3c); however, the side chains adopt different conformations in the two structures.

Importantly, overlays of the bicyclic portions of the tricyclic TAN core with the bicyclic TAN reveals a steric clash for a potential tricycle, including its side chain, at the $\mathrm{AmpC}_{E C}$ active site (Figure 4). This analysis is consistent with the observed bicyclic structure at the $\mathrm{AmpC}_{E C}$ active site and indicates that it is unlikely that the boronates studied can bind to the $\mathrm{AmpC}_{E C}$ active site in the tricyclic form (at least without conformational change). Further comparisons suggest that the other SBLs, for which crystallographic studies are reported in complex with the bicyclic form of TAN (i.e., KPC-2 [31] (PDB ID 6TD1), CTX-M-15 [26] (PDB ID 6SP6), OXA-10 [24] (PDB ID 6RTN)), will also not accommodate the tricyclic core, assuming a conserved general binding mode of the bicycle and no conformational changes (Figure S5).

(a)

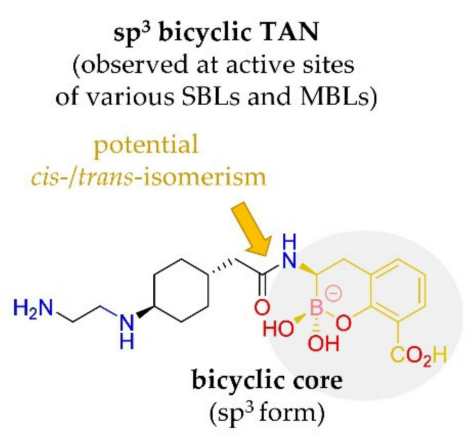

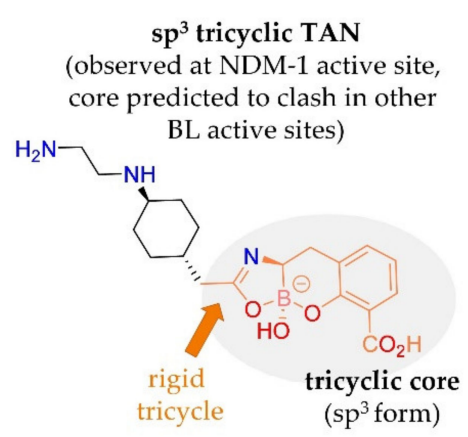
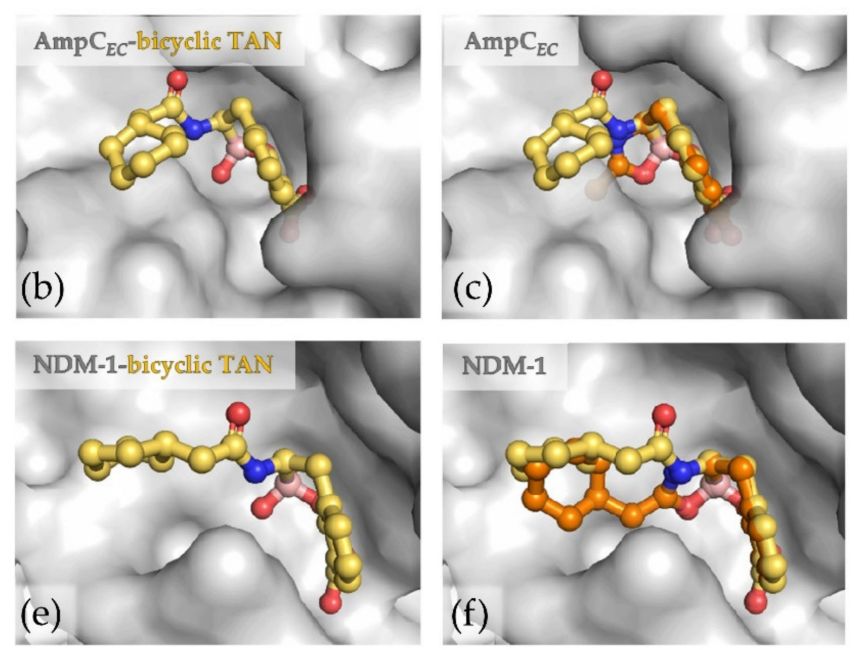

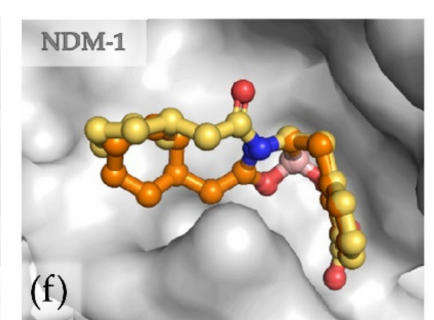

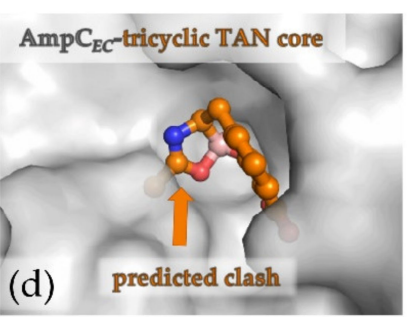

(d)

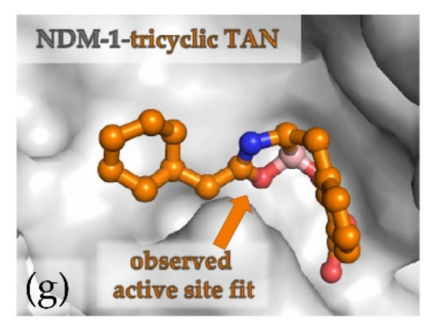

Figure 4. Tricyclic TAN likely cannot bind at the $A m p C_{E C}$ active site due to a steric clash of the putative tricyclic core and side chain. (a) Structural comparison of tricyclic and bicyclic forms of TAN; (b-g) overlay of tricyclic TAN core (orange, not crystallographically observed at $\mathrm{AmpC}_{E C}$ active site) with bicyclic TAN (yellow) crystallographically observed bound to active site S64 of AmpC $\mathrm{AC}_{\text {(PDB }}$ ID: 6YEN) reveals a likely steric clash of the rigid tricycle in the $\mathrm{AmpC}_{E C}$ active site as well as active sites of other $\beta$-lactamases (Figure S5). By contrast, both tricyclic (orange) and bicyclic (yellow) forms of TAN have been crystallographically observed at the active site of the B1 MBL NDM-1 (PDB ID: 6RMF) [24]. Note, in both structures, that the terminal amine of the side chain was disordered and therefore excluded from the model; (b) observed conformation of bicyclic TAN at the AmpC $E C$ active site; (c) alignment of tricyclic TAN core to bicyclic TAN observed at the $A m p C_{E C}$ active site; (d) putative steric clash of tricyclic TAN in the $\mathrm{AmpC}_{E C}$ active site based on the overlay in c). Note the apparently flexible parts of the side chain are not shown, but would make a clear steric clash with the active site; (e) observed conformation of bicyclic TAN at the NDM-1 active site; (f) overlay of tricyclic TAN and bicyclic TAN, both as observed at the NDM-1 active site; (g) observed conformation of tricyclic TAN at the NDM-1 active site. 
Although there are substantial differences in the active site chemistry of the SBLs and MBLs, crystallographic analysis on the class B1 MBL VIM-2 [26] (PDB ID: 6SP7) shows that the bicyclic conformation of TAN as observed in SBLs is maintained in binding to this clinically relevant MBL (Figure S5). In the case of NDM-1, active site binding of both bicyclic and tricyclic TAN was observed as shown in Figure $4 \mathrm{e}-\mathrm{g}$ ( $~ 75 \%$ of the active sites were occupied by the tricyclic and $\sim 25 \%$ by the bicyclic form) [24]. By contrast, overlay of the tricyclic form of TAN with the bicyclic form of TAN as observed in the active site of VIM-2 reveals a steric clash (Figure S5). This implies that the tricyclic form of TAN is likely relevant to the inhibition of some (NDM-1), but not all MBLs, assuming a conserved binding mode for the tri-/bi-cycle core and a lack of substantial conformational changes. It should be noted that recent work has shown other tricyclic boronates, i.e., those containing a cyclopropyl group fused to the C2/C3 carbons of the bicyclic boronate potently inhibit both SBLs and MBLs [50], though the cyclopropyl ring methylene projects in a different orientation to the 'third' ring of the tricyclic ring system that can be formed with TAN (Figure S6).

\section{Conclusions}

Following the clinical application of boronic acids as proteasome inhibitors for treatment of myeloma, boronic acids/boronates have become more common in medicinal chemistry [17]. In the antibacterial field, the monocyclic boronate vaborbactam has been approved for clinical use as an inhibitor of class A and some class C SBLs [19]. More recent work has demonstrated that the scope of boronates as $\beta$-lactamase inhibitors can be extended to multiple SBLs and MBLs by integration into a bicyclic scaffold [20,21], as present in TAN [26] (Figure 1d), which is currently in late stage clinical trials. Our kinetic and structural studies with TAN and other bicyclic boronate derivatives (CB2, CB3) with different C-3 side chains reveal their potential for inhibition of the class C AmpC serine- $\beta$-lactamase from Escherichia coli.

Our results further support the potential of bicyclic boronates as unusually broad-spectrum inhibitors of all SBL types and, at least B1 type, MBLs. It is possible that the potential of cyclic boronates as clinically useful inhibitors of two or more mechanistically distinct groups of enzymes will extend to other families, such as proteases. Improvements in methods for their preparation should enable more systematic SAR. In this regard, it may be that the incorporation of the boron into a ring system that limits conformations and modes of reactivity may both enhance potency and reduce unwanted modes of reaction (e.g., oligomerization) compared to acyclic boronic acids.

Combined with previous reports, our three crystal structures of bicyclic boronates with $\mathrm{AmpC}_{E C}$ reveal remarkable similarity between the binding modes of the 6,6-core ring system of the bicyclic boronates to SBLs from class A (e.g., CTX-M-15 [21,26], L2 [33], and KPC-2 [31]), class D (e.g., OXA-10 [20,24]) and the class C AmpC from Pseudomonas aeruginosa [32] as well as the penicillin-binding protein-5 (PBP-5) from Escherichia coli [20]. The results thus support the proposal that mimicking high-energy intermediates common to different types of enzymes can enable their inhibition by the same inhibitor. Note that the reported bicyclic boronates tend to be weaker PBP inhibitors (and hence antibiotics) than SBL/B1 MBL inhibitors [20,21]; this may reflect the fact that, although they react with $\beta$-lactams, PBPs are not optimized to catalyze their hydrolysis. Alternatively, it may be that optimized functionalization of the core bicyclic boronate will enable potent PBP inhibition. The combined results show that, in the case of SBL/MBL inhibition by bicyclic boronates, there is scope for (C-3) side chain optimization, which may further broaden the scope of $\beta$-lactamase inhibition, including to multiple B2/B3 type MBLs. Optimization in potency will need to be carried out in parallel with that at a microbiological level, as has been done for TAN with respect to improved cell penetration [26]. Interestingly, although VAB was substantially less potent than TAN against isolated $\mathrm{AmpC}_{E C}$, it performed equally well against our AmpC bearing engineered E. coli strain as TAN (Table 2), possibly reflecting either improved penetration or reduced efflux.

Together with previous reports showing enhanced activity of CB3 against class D SBLs, as well as certain B2 and B3 MBLs compared to TAN and CB2 [16], our results suggest the thioether CB3 could be 
a promising start for further optimization towards a broad-spectrum, dual SBL and MBL inhibitor. Selective binding of the minor (3S)-isomer indicates that stereoselective synthesis might further benefit $\mathrm{AmpC}_{E C}$ inhibition. Further optimization will need to address the relatively low potency of CB3 in cells, which could reflect potential poor cell penetration of CB3 due to its lipophilicity. CB3 has a predicted $\log \mathrm{P}$ of 4.42 ; studies have shown that compounds with relatively low $\operatorname{logPs}(\mathrm{e} . \mathrm{g}$., CB2, TAN and VAB with predicted $\log$ Ps of $2.09,1.50$, and 1.86 , respectively) manifest improved penetration of Gram-negative bacteria [51], leaving further scope for optimization of the thioether side chain, e.g., by addition of an amino group as in TAN [26].

The observation of an apparently weakly bound tricyclic form of TAN on the surface of $A m p C_{E C}$ is of interest from a boronate chemistry perspective as it potentially further exemplifies the ability of boron-based inhibitors to interchange between different forms in aqueous solution. It should be noted that, in both the cases where a tricyclic boronate form has been observed crystallographically, i.e., with $\mathrm{AmpC}_{E C}$ and the MBL NDM-1, the crystallization conditions were acidic (pH 6.0 and 5.8 [24], respectively). Thus, the tricycle could be formed in aqueous solution during crystallization; such formation in aqueous solution is precedented in small molecule chemistry [49]. Comparison of SBL and MBL structures with tri- and bi-cyclic boronates suggests most SBL active sites will not readily accommodate tricylic structures of the type reported here and previously [24]. Although tricyclic TAN likely does not significantly contribute to inhibition of $\mathrm{AmpC}_{E C}$ and related SBLs, at least one $\beta$-lactamase (NDM-1) can bind a tricyclic form at its active site (at least in crystals) [24], thus the ability of boronate type inhibitors to interchange between different forms has the potential to extend the range of $\beta$-lactamases usefully inhibited by them.

Supplementary Materials: The following are available online at http://www.mdpi.com/2218-273X/10/6/899/s1, Table S1: Data collection and refinement statistics of $\mathrm{AmpC}_{E C}$ crystals. Figure S1: Kinetic characterization of reversible $\mathrm{AmpC}_{E C}$ inhibition by cyclic boronates. Figure S2: Views from a crystal Structure of $\mathrm{AmpC}_{E C}$ in complex with CB2. Figure S3: Views from a crystal structure of $\mathrm{AmpC}_{E C}$ in complex with TAN. Figure S4: Views from a crystal structure of $\mathrm{AmpC}_{E C}$ in complex with CB3. Figure S5: The tricyclic form of TAN likely cannot bind to some $\beta$-lactamases including members from all Ambler classes. Figure S6: Structures of the tricyclic form of TAN and QPX7728.

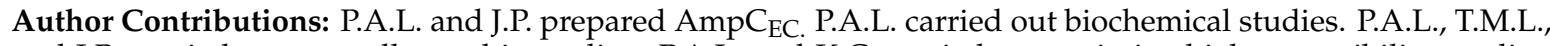
and J.B. carried out crystallographic studies. P.A.L. and K.C. carried out antimicrobial susceptibility studies. A.P. prepared CB3. R.C. prepared CB2. A.K. with the help of T.D.P. prepared TAN. C.W.G.F., M.G.P.P., P.T., C.J.S., and J.B. supervised the work. P.A.L. drafted the original version of the manuscript with assistance of A.P. All authors have read and agreed to the submitted version of the manuscript.

Funding: P.A.L. thanks the National PhD Training Programme in Antimicrobial Resistance Research by the Medical Research Foundation (MRF-145-0004-TPG-AVISO) for funding a postgraduate studentship. This research was further funded by the Wellcome Trust, the Medical Research Council, the Biotechnology and Biological Research Council (BB/S50676X/1), and the Innovative Medicines Initiative (European Lead factory and ENABLE components).

Acknowledgments: Diffraction data were collected at the I03 and I24 beamlines at the Diamond Light Source, Didcot, United Kingdom.

Conflicts of Interest: The authors declare no conflict of interest.

\section{References}

1. Versporten, A.; Bolokhovets, G.; Ghazaryan, L.; Abilova, V.; Pyshnik, G.; Spasojevic, T.; Korinteli, I.; Raka, L.; Kambaralieva, B.; Cizmovic, L.; et al. Antibiotic use in eastern Europe: A cross-national database study in coordination with the WHO Regional Office for Europe. Lancet Infect. Dis. 2014, 14, 381-387. [CrossRef]

2. Bush, K. Past and Present Perspectives on $\beta$-Lactamases. Antimicrob. Agents Chemother. 2018, 62, e01076-18. [CrossRef] [PubMed]

3. Drawz, S.M.; Bonomo, R.A. Three decades of $\beta$-lactamase inhibitors. Clin. Microbiol. Rev. 2010, $23,160-201$. [CrossRef] [PubMed]

4. Bush, K.; Jacoby, G.A. Updated Functional Classification of $\beta$-Lactamases. Antimicrob. Agents Chemother. 2010, 54, 969-976. [CrossRef] [PubMed] 
5. Leigh, D.A.; Bradnock, K.; Marriner, J.M. Augmentin (amoxycillin and clavulanic acid) therapy in complicated infections due to $\beta$-lactamase producing bacteria. J. Antimicrob. Chemother. 1981, 7, 229-236. [CrossRef]

6. Reading, C.; Cole, M. Clavulanic acid: A $\beta$-lactamase-inhiting $\beta$-lactam from Streptomyces clavuligerus. Antimicrob. Agents Chemother. 1977, 11, 852-857. [CrossRef] [PubMed]

7. Benson, J.M.; Nahata, M.C. Sulbactam/ampicillin, a new $\beta$-lactamase inhibitor/ $\beta$-lactam antibiotic combination. Drug Intell. Clin. Pharm. 1988, 22, 534-541. [CrossRef]

8. Gutmann, L.; Kitzis, M.D.; Yamabe, S.; Acar, J.F. Comparative evaluation of a new $\beta$-lactamase inhibitor, YTR 830, combined with different $\beta$-lactam antibiotics against bacteria harboring known $\beta$-lactamases. Antimicrob. Agents Chemother. 1986, 29, 955-957. [CrossRef]

9. Blizzard, T.A.; Chen, H.; Kim, S.; Wu, J.; Bodner, R.; Gude, C.; Imbriglio, J.; Young, K.; Park, Y.-W.; Ogawa, A.; et al. Discovery of MK-7655, a $\beta$-lactamase inhibitor for combination with Primaxin ${ }^{\circledR}$. Bioorg. Med. Chem. Lett. 2014, 24, 780-785. [CrossRef]

10. Wang, D.Y.; Abboud, M.I.; Markoulides, M.S.; Brem, J.; Schofield, C.J. The road to avibactam: The first clinically useful non- $\beta$-lactam working somewhat like a $\beta$-lactam. Future Med. Chem. 2016, 8, 1063-1084. [CrossRef]

11. Ehmann, D.E.; Jahic, H.; Ross, P.L.; Gu, R.-F.; Hu, J.; Durand-Réville, T.F.; Lahiri, S.; Thresher, J.; Livchak, S.; Gao, N.; et al. Kinetics of avibactam inhibition against Class A, C, and D $\beta$-lactamases. J. Biol. Chem. 2013, 288, 27960-27971. [CrossRef] [PubMed]

12. Lahiri, S.D.; Walkup, G.K.; Whiteaker, J.D.; Palmer, T.; McCormack, K.; Tanudra, M.A.; Nash, T.J.; Thresher, J.; Johnstone, M.R.; Hajec, L.; et al. Selection and molecular characterization of ceftazidime/avibactam-resistant mutants in Pseudomonas aeruginosa strains containing derepressed AmpC. J. Antimicrob. Chemother. 2015, 70, 1650-1658. [CrossRef] [PubMed]

13. Shields, R.K.; Chen, L.; Cheng, S.; Chavda, K.D.; Press, E.G.; Snyder, A.; Pandey, R.; Doi, Y.; Kreiswirth, B.N.; Nguyen, M.H.; et al. Emergence of Ceftazidime-Avibactam Resistance Due to Plasmid-Borne $b l a_{\mathrm{KPC}-3}$ Mutations during Treatment of Carbapenem-Resistant Klebsiella pneumoniae Infections. Antimicrob. Agents Chemother. 2017, 61, 1-11. [PubMed]

14. Abboud, M.I.; Damblon, C.; Brem, J.; Smargiasso, N.; Mercuri, P.; Gilbert, B.; Rydzik, A.M.; Claridge, T.D.; Schofield, C.J.; Frere, J.M. Interaction of Avibactam with Class B Metallo- $\beta$-Lactamases. Antimicrob. Agents Chemother. 2016, 60, 5655-5662. [CrossRef]

15. Pogue, J.M.; Bonomo, R.A.; Kaye, K.S. Ceftazidime/Avibactam, Meropenem/Vaborbactam, or Both? Clinical and Formulary Considerations. Clin. Infect. Dis. 2019, 68, 519-524. [CrossRef] [PubMed]

16. Parkova, A.; Lucic, A.; Krajnc, A.; Brem, J.; Calvopiña, K.; Langley, G.W.; McDonough, M.A.; Trapencieris, P.; Schofield, C.J. Broad Spectrum $\beta$-Lactamase Inhibition by a Thioether Substituted Bicyclic Boronate. ACS Infect. Dis. 2019. [CrossRef] [PubMed]

17. Krajnc, A.; Lang, P.A.; Panduwawala, T.D.; Brem, J.; Schofield, C.J. Will morphing boron-based inhibitors beat the $\beta$-lactamases? Curr. Opin. Chem. Biol. 2019, 50, 101-110. [CrossRef] [PubMed]

18. Hecker, S.J.; Reddy, K.R.; Totrov, M.; Hirst, G.C.; Lomovskaya, O.; Griffith, D.C.; King, P.; Tsivkovski, R.; Sun, D.; Sabet, M.; et al. Discovery of a Cyclic Boronic Acid $\beta$-Lactamase Inhibitor (RPX7009) with Utility vs Class A Serine Carbapenemases. J. Med. Chem. 2015, 58, 3682-3692. [CrossRef] [PubMed]

19. Jorgensen, S.C.J.; Rybak, M.J. Meropenem and Vaborbactam: Stepping up the Battle against Carbapenem-resistant Enterobacteriaceae. Pharmacotherapy 2018, 38, 444-461. [CrossRef]

20. Brem, J.; Cain, R.; Cahill, S.; McDonough, M.A.; Clifton, I.J.; Jimenez-Castellanos, J.C.; Avison, M.B.; Spencer, J.; Fishwick, C.W.; Schofield, C.J. Structural basis of metallo- $\beta$-lactamase, serine- $\beta$-lactamase and penicillin-binding protein inhibition by cyclic boronates. Nat. Commun. 2016, 7, 12406. [CrossRef]

21. Cahill, S.T.; Cain, R.; Wang, D.Y.; Lohans, C.T.; Wareham, D.W.; Oswin, H.P.; Mohammed, J.; Spencer, J.; Fishwick, C.W.; McDonough, M.A.; et al. Cyclic Boronates Inhibit All Classes of $\beta$-Lactamases. Antimicrob. Agents Chemother. 2017, 61, 1-13. [CrossRef]

22. Geibel, B.; Dowell, J.; Dickerson, D.; Henkel, T. 1401. A Randomized, Double-Blind, Placebo-Controlled Study of the Safety and Pharmacokinetics of Single and Repeat Doses of VNRX-5133 in Healthy Subjects. Open Forum Infect. Dis. 2018, 5 (Suppl. 1), S431. [CrossRef] 
23. Wang, X.; Zhao, C.; Wang, Q.; Wang, Z.; Liang, X.; Zhang, F.; Zhang, Y.; Meng, H.; Chen, H.; Li, S.; et al. In vitro activity of the novel $\beta$-lactamase inhibitor taniborbactam (VNRX-5133), in combination with cefepime or meropenem, against MDR Gram-negative bacterial isolates from China. J. Antimicrob Chemother. 2020. [CrossRef] [PubMed]

24. Krajnc, A.; Brem, J.; Hinchliffe, P.; Calvopina, K.; Panduwawala, T.D.; Lang, P.A.; Kamps, J.; Tyrrell, J.M.; Widlake, E.; Saward, B.G.; et al. Bicyclic Boronate VNRX-5133 Inhibits Metallo- and Serine- $\beta$-Lactamases. J. Med. Chem. 2019, 62, 8544-8556. [CrossRef] [PubMed]

25. Hamrick, J.C.; Docquier, J.D.; Uehara, T.; Myers, C.L.; Six, D.A.; Chatwin, C.L.; John, K.J.; Vernacchio, S.F.; Cusick, S.M.; Trout, R.E.L.; et al. VNRX-5133 (Taniborbactam), a Broad-Spectrum Inhibitor of Serineand Metallo- $\beta$-Lactamases, Restores Activity of Cefepime in Enterobacterales and Pseudomonas aeruginosa. Antimicrob. Agents Chemother. 2020, 64, 1-19. [CrossRef] [PubMed]

26. Liu, B.; Trout, R.E.L.; Chu, G.-H.; McGarry, D.; Jackson, R.W.; Hamrick, J.C.; Daigle, D.M.; Cusick, S.M.; Pozzi, C.; De Luca, F.; et al. Discovery of Taniborbactam (VNRX-5133): A Broad-Spectrum Serine- and Metallo- $\beta$-lactamase Inhibitor for Carbapenem-Resistant Bacterial Infections. J. Med. Chem. 2020, 63, 2789-2801. [CrossRef]

27. Jacoby, G.A. AmpC $\beta$-Lactamases. Clin. Microbiol. Rev. 2009, 22, 161-182. [CrossRef]

28. Jaurin, B.; Grundström, T.; Edlund, T.; Normark, S. The E. coli $\beta$-lactamase attenuator mediates growth rate-dependent regulation. Nature 1981, 290, 221-225. [CrossRef] [PubMed]

29. Nelson, E.C.; Elisha, B.G. Molecular Basis of AmpC Hyperproduction in Clinical Isolates of Escherichia coli. Antimicrob. Agents Chemother. 1999, 43, 957-959. [CrossRef] [PubMed]

30. Vila, J.; Sáez-López, E.; Johnson, J.R.; Römling, U.; Dobrindt, U.; Cantón, R.; Giske, C.G.; Naas, T.; Carattoli, A.; Martínez-Medina, M.; et al. Escherichia coli: An old friend with new tidings. FEMS Microbiol. Rev. 2016, 40, 437-463. [CrossRef] [PubMed]

31. Tooke, C.L.; Hinchliffe, P.; Krajnc, A.; Mulholland, A.J.; Brem, J.; Schofield, C.J.; Spencer, J. Cyclic boronates as versatile scaffolds for KPC-2 $\beta$-lactamase inhibition. RCS Med. Chem. 2020, 11, 491-496. [CrossRef]

32. Cahill, S.T.; Tyrrell, J.M.; Navratilova, I.H.; Calvopina, K.; Robinson, S.W.; Lohans, C.T.; McDonough, M.A.; Cain, R.; Fishwick, C.W.G.; Avison, M.B.; et al. Studies on the inhibition of AmpC and other $\beta$-lactamases by cyclic boronates. Biochim. Biophys. Acta Gen. Subj. 2019, 1863, 742-748. [CrossRef] [PubMed]

33. Calvopiña, K.; Hinchliffe, P.; Brem, J.; Heesom, K.J.; Johnson, S.; Cain, R.; Lohans, C.T.; Fishwick, C.W.G.; Schofield, C.J.; Spencer, J.; et al. Structural/mechanistic insights into the efficacy of nonclassical $\beta$-lactamase inhibitors against extensively drug resistant Stenotrophomonas maltophilia clinical isolates. Mol. Microbiol. 2017, 106, 492-504. [CrossRef] [PubMed]

34. Burns, C.J. Beta-lactamase inhibitors. PCT Patent WO 2010/130708 A1, 18 November 2010.

35. Reddy, R.K. Boronic acid derivatives and therapeutic uses thereof. PCT Patent WO 2016003929 A1, 7 January 2016.

36. Van Berkel, S.S.; Brem, J.; Rydzik, A.M.; Salimraj, R.; Cain, R.; Verma, A.; Owens, R.J.; Fishwick, C.W.G.; Spencer, J.; Schofield, C.J. Assay platform for clinically relevant metallo- $\beta$-lactamases. J. Med. Chem. 2013, 56, 6945-6953. [CrossRef] [PubMed]

37. Page, M.G. The kinetics of non-stoichiometric bursts of $\beta$-lactam hydrolysis catalysed by class $C \beta$-lactamases. Biochem. J. 1993, 295 Pt 1, 295-304. [CrossRef]

38. Dubus, A.; Wilkin, J.M.; Raquet, X.; Normark, S.; Frère, J.M. Catalytic mechanism of active-site serine $\beta$-lactamases: Role of the conserved hydroxy group of the Lys-Thr(Ser)-Gly triad. Biochem. J. 1994, 301 Pt 2, 485-494. [CrossRef]

39. Morrison, J.F.; Walsh, C.T. The behavior and significance of slow-binding enzyme inhibitors. Adv. Enzymol. Rel. Areas Mol. Biol. 1988, 61, 201-301.

40. Williams, J.W.; Morrison, J.F. The kinetics of reversible tight-binding inhibition. In Methods Enzymology; Academic Press: Cambridge, MA, USA, 1979; Volume 63, pp. 437-467.

41. Copeland, R.A.; Basavapathruni, A.; Moyer, M.; Scott, M.P. Impact of enzyme concentration and residence time on apparent activity recovery in jump dilution analysis. Anal. Biochem. 2011, 416, 206-210. [CrossRef]

42. McCoy, A.J.; Grosse-Kunstleve, R.W.; Adams, P.D.; Winn, M.D.; Storoni, L.C.; Read, R.J. Phaser crystallographic software. J. Appl. Crystallogr. 2007, 40 Pt 4, 658-674. [CrossRef] 
43. Powers, R.A.; Caselli, E.; Focia, P.J.; Prati, F.; Shoichet, B.K. Structures of Ceftazidime and Its Transition-State Analogue in Complex with AmpC $\beta$-Lactamase: Implications for Resistance Mutations and Inhibitor Design. Biochemistry 2001, 40, 9207-9214. [CrossRef]

44. Adams, P.D.; Grosse-Kunstleve, R.W.; Hung, L.W.; Ioerger, T.R.; McCoy, A.J.; Moriarty, N.W.; Read, R.J.; Sacchettini, J.C.; Sauter, N.K.; Terwilliger, T.C. PHENIX: Building new software for automated crystallographic structure determination. Acta Crystallogr. D Biol. Crystallogr. 2002, 58 Pt 11, 1948-1954. [CrossRef] [PubMed]

45. Emsley, P.; Lohkamp, B.; Scott, W.G.; Cowtan, K. Features and development of Coot. Acta Crystallogr. D Biol. Crystallogr. 2010, 66 Pt 4, 486-501. [CrossRef] [PubMed]

46. Lahiri, S.D.; Mangani, S.; Durand-Reville, T.; Benvenuti, M.; De Luca, F.; Sanyal, G.; Docquier, J.D. Structural insight into potent broad-spectrum inhibition with reversible recyclization mechanism: Avibactam in complex with CTX-M-15 and Pseudomonas aeruginosa AmpC $\beta$-lactamases. Antimicrob. Agents Chemother. 2013, 57, 2496-2505. [CrossRef] [PubMed]

47. Lahiri, S.D.; Johnstone, M.R.; Ross, P.L.; McLaughlin, R.E.; Olivier, N.B.; Alm, R.A. Avibactam and class C $\beta$-lactamases: Mechanism of inhibition, conservation of the binding pocket, and implications for resistance. Antimicrob. Agents Chemother. 2014, 58, 5704-5713. [CrossRef]

48. Haenni, M.; Châtre, P.; Madec, J.-Y. Emergence of Escherichia coli producing extended-spectrum AmpC $\beta$-lactamases (ESAC) in animals. Front. Microbiol. 2014, 5, 53. [CrossRef]

49. Inglis, S.R.; Woon, E.C.Y.; Thompson, A.L.; Schofield, C.J. Observations on the Deprotection of Pinanediol and Pinacol Boronate Esters via Fluorinated Intermediates. J. Org. Chem. 2010, 75, 468-471. [CrossRef]

50. Hecker, S.J.; Reddy, K.R.; Lomovskaya, O.; Griffith, D.C.; Rubio-Aparicio, D.; Nelson, K.; Tsivkovski, R.; Sun, D.; Sabet, M.; Tarazi, Z.; et al. Discovery of Cyclic Boronic Acid QPX7728, an Ultrabroad-Spectrum Inhibitor of Serine and Metallo- $\beta$-lactamases. J. Med. Chem. 2020. [CrossRef]

51. Ebejer, J.-P.; Charlton, M.H.; Finn, P.W. Are the physicochemical properties of antibacterial compounds really different from other drugs? J. Cheminform. 2016, 8, 30. [CrossRef]

(C) 2020 by the authors. Licensee MDPI, Basel, Switzerland. This article is an open access article distributed under the terms and conditions of the Creative Commons Attribution (CC BY) license (http://creativecommons.org/licenses/by/4.0/). 